\title{
Sporadic Creutzfeldt-Jakob disease prion infection of human cerebral organoids
}

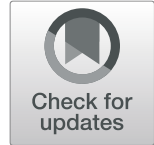

Bradley R. Groveman', Simote T. Foliaki ${ }^{1}$, Christina D. Orru', Gianluigi Zanusso ${ }^{2}$, James A. Carroll', Brent Race ${ }^{1}$ and Cathryn L. Haigh ${ }^{*}$ (i)

\begin{abstract}
For the transmissible, neurogenerative family of prion diseases, few human models of infection exist and none represent structured neuronal tissue. Human cerebral organoids are self-organizing, three-dimensional brain tissues that can be grown from induced pluripotent stem cells. Organoids can model aspects of neurodegeneration in Alzheimer's Disease and Down's Syndrome, reproducing tau hyperphosphorylation and amyloid plaque pathology. To determine whether organoids could be used to reproduce human prion infection and pathogenesis, we inoculated organoids with two sporadic Creutzfeldt-Jakob Disease prion subtypes. Organoids showed uptake, followed by clearance, of the infectious inoculum. Subsequent re-emergence of prion self-seeding activity indicated de novo propagation. Organoid health assays, prion titer, prion protein electrophoretic mobility and immunohistochemistry demonstrated inoculum-specific differences. Our study shows, for the first time, that cerebral organoids can model aspects of human prion disease and thus offer a powerful system for investigating different human prion subtype pathologies and testing putative therapeutics.
\end{abstract}

Keywords: Prion, CJD, Human cerebral organoid, Induced pluripotent stem cells, RT-QulC

\section{Introduction}

The transmissible, neurodegenerative prion diseases affect humans and animals. Acquired prion diseases arise due to exposure to prion contaminated material. However, prion diseases more often occur sporadically or due to hereditary mutations within the gene encoding the prion protein (PrP). In cases of human sporadic Creutzfeldt-Jakob Disease (sCJD), different molecular subtypes of prions have been recognized $[4,13,16,32,33]$ that influence the clinical and pathological disease phenotypes. These subtypes are identified by the electrophoretic mobility pattern of the disease-associated, protease-resistant prion protein $\left(\mathrm{PrP}^{\mathrm{Res}}\right)$ and by a genetic polymorphism at amino acid residue 129 , which may be a methionine or valine. The heterogeneity of the human subtypes and the brain regions they target [39] causes complications when attempting to model human diseases in cell models as homogenous cell systems cannot address different neuronal vulnerabilities.

\footnotetext{
*Correspondence: cathryn.haigh@nih.gov

'Division of Intramural Research, Laboratory of Persistent Viral Diseases,

National Institute of Allergy and Infectious Diseases, Rocky Mountain

Laboratories, National Institutes of Health, 903 South 4th Street, Hamilton, MT 59840, USA

Full list of author information is available at the end of the article
}

There are further challenges associated with investigating human prion diseases in vitro. First and foremost, human cell lines have traditionally proven refractive to prion infection. Only two cell models of prion infection and propagation have been described that express normal human PrP levels in a human cellular background. The first, published in 1995, reported stable CJD infection in sub-clones from the Sh-SY5Y neuroblastoma cell line [19] but no further use of this model has ever been reported. Recently, the second showed that astrocytes differentiated from human induced pluripotent stem cells (hu-iPSCs) can take up and propagate prion infection from sCJD samples [18]. Uptake of infection by these astrocytes was influenced by SCJD subtype and residue 129 polymorphism ( $\mathrm{M}$ or $\mathrm{V}$ ) of both the host cells and the inocula. This is the first fully human cell model developed since the 1995 Sh-SY5Y study, and the only human model of infection currently reported. Despite the significant advancement this model offers, it does not allow for studying how human neurons respond to infection or for investigating potential three-dimensional cellular cross-talk.

$\mathrm{Hu}$-iPSCs have also been used to produce three-dimensional, highly organized cerebral tissues; referred to as

(c) The Author(s). 2019 Open Access This article is distributed under the terms of the Creative Commons Attribution 4.0 International License (http://creativecommons.org/licenses/by/4.0/), which permits unrestricted use, distribution, and 
cerebral organoids [20, 35]. Cerebral organoids display self-organization that generates an organ-like configuration and, following the development of cortex neuronal populations, also populate with astrocytes and oligodendrocytes creating the closest in vitro model of human brain tissue yet described [20, 25, 35]. Organoids also develop functional electrical signaling and can survive in culture for many months; some may survive years [20,44]. Cerebral organoids have demonstrated substantial potential for modelling developmental diseases, such as the microcephaly associated with Zika virus infection [21, 38]. Plus, their longevity permits their use for investigating brain diseases that develop over a longer time frame. For example, organoids exhibit the tau hyperphosphorylation plus amyloid deposition associated with Alzheimer's Disease and Down's syndrome dementia [14].

Organoids or neuronal cultures from hu-iPSCs have similarly been used to model prion dysfunction in cells from people who are predisposed to developing prion disease due to mutations within the prion gene $[14,26]$. Organoids generated from a patient carrying a Y218N PrP mutation (that causes Gerstmann-Sträussler-Scheinker prion disease) were able to reproduce certain pathological changes, such as astrogliosis and tau hyperphosphorylation, but did not replicate $\operatorname{PrP}^{\text {Res }}$ production [26]. We hypothesized that, in addition to their use modeling genetic prion diseases, cerebral organoid cultures could be used to develop an in vitro human model of prion infection in organized neuronal tissue by exposing them to sCJD prions. In this study we found that human cerebral organoids are susceptible to prion infection. Uptake of infection was influenced by the infecting inocula and the de novo prions produced were influenced by the underlying organoid. We conclude that human cerebral organoids offer a new three-dimensional, neuronal tissue model of human prion disease.

\section{Materials and methods}

Human induced pluripotent stem cells and culture KYOU-DXR0109B (ACS-1023; ATCC) hu-iPSCs were routinely cultured on low growth factor Matrigel (Roche) in mTeSR1 medium (Stem Cell Technologies) with 5\% $\mathrm{CO}_{2}$ in a humidified incubator as described in the mTeSR handbook. Colonies were passaged at approximately $70-80 \%$ confluency before colonies had started to contact each other.

\section{Human cerebral organoid generation and routine culture}

Cerebral organoids were generated as described in Lancaster and Knoblich [20]. The following variations to the protocol were made: For embryoid body $(\mathrm{EB})$ production one $25 \mathrm{~cm}^{2}$ flask of hu-iPSCs was used to seed a 96-well plate. Cells were plated in $100 \mu \mathrm{l} \mathrm{hES} \mathrm{medium} \mathrm{(20 \%} \mathrm{[v/v]}$ knock-out serum replacement, $0.03 \%$ [v/v] fetal bovine serum, $1 \times$ glutamax, $1 \times$ non-essential amino acids, and $1 \mu \mathrm{l} / 142.86 \mathrm{ml}$ 2-Merceptoethanol in DME-F12 medium, additionally supplemented on day 0 with $50 \mu \mathrm{M}$ Y27632 and $4 \mathrm{ng} / \mathrm{ml} \mathrm{rh}$ FGF), with a further $100 \mu \mathrm{l}$ added at days 2 and 4 . EBs were transferred into neural induction medium (1× glutamax, $1 \times$ non-essential amino acids, $1 \%[\mathrm{v} / \mathrm{v}] \mathrm{N} 2$, and $1 \mu \mathrm{g} / \mathrm{ml}$ heparin in DME-F12 medium) in low adhesion plates between days 4 and 6 depending upon morphology. Organoids were embedded 2-4 days after neural induction when neuroecotoderm was visible. Matrigel embedding was done in a 6-well plate with 6 organoids per well using $30 \mu \mathrm{l}$ of Matrigel (Roche) and incubated in cerebral organoid media $(1 \times$ glutamax, $1 \times$ penicillin-streptomycin solution, $0.5 \times$ non-essential amino acids, $0.5 \%[\mathrm{v} / \mathrm{v}] \mathrm{N} 2,1 \mu \mathrm{l} / 4 \mathrm{ml}$ insulin, and $1 \mu \mathrm{l} / 286 \mathrm{ml}$ 2-Merceptoethanol in 1:1 Neurobasal:DME-F12 medium) with 1\% (v/v) B12 minus retinoic acid. On day four, organoids were transferred into cerebral organoid medium with $1 \%(\mathrm{v} / \mathrm{v})$ B12 plus retinoic acid for long-term culture. Long-term agitated culture was performed in upright $25 \mathrm{~cm}^{2}$ low-adhesion flasks on an orbital shaker at $85 \mathrm{rpm}$. Media was changed twice weekly.

\section{Prion infections of human cerebral organoids}

Brain homogenates from sporadic CJD subtypes MV1 and MV2 were diluted into organoid maintenance media to a final concentration of $0.1 \%$ (tissue wet weight/volume). The inoculated media was filter sterilized through a 0.22 nitrocellulose filter. To ensure the infectious seeds within the inoculum had not been lost in the filtering process, RT-QuIC analysis (described below) was performed on the media. Significant RT-QuIC seeding activity was found in both MV1 and MV2 inocula (Table 1). Organoids were distributed between 6-well plates (3 organoids per well) for Prestoblue and LDH monitoring and T25 flasks for routine maintenance (6-12 organoids/flask). At the start of infection, existing media was removed from the organoids and replaced with the inoculated media. Twenty-four hours after inoculation an equivalent volume of fresh media was added to the cultures (diluting the original inoculum 1 in 2). Organoids were incubated for a further 3 days before a half media exchange. A full media and culture vessel exchange was performed 7 days after initial exposure. Organoids were maintained in agitated culture with three media changes per week. All brain tissues used in this study were obtained on autopsy and were therefore exempt from review by the NIH Office of Human Subjects Research Protections.

Table 1 sCJD inoculum information

\begin{tabular}{lllll}
\hline Inocula & Gender & $\begin{array}{l}\text { Age at onset } \\
\text { (years) }\end{array}$ & $\begin{array}{l}\text { Disease duration } \\
\text { (months) }\end{array}$ & Inoculum SD $50 / \mu \mathrm{l}$ \\
\hline MV1 & $M$ & 62 & 14 & $1.58 \times 10^{5}$ \\
MV2 & $M$ & 57 & 15 & $8.89 \times 10^{5}$ \\
\hline
\end{tabular}




\section{Prestoblue analysis}

Prestoblue metabolism was measured as per the manufacturer's instructions once weekly or as required (the same 3 organoids were monitored from start to finish of the cultures). Briefly, Prestoblue reagent was diluted 1 in 10 in organoid media. Existing organoid media was removed and organoids were incubated in Prestoblue-containing media for 30 mins. The metabolized Prestoblue containing media was then transferred into replicate wells for analysis. Prestoblue fluorescence was measured at $560 \mathrm{~nm}$ excitation and $590 \mathrm{~nm}$ emission in a ClarioStar plate reader (BMG).

\section{Lactate Dyhydrogenase (LDH) analysis}

Extracellular LDH was measured using cytotoxicity detection kit plus [LDH] (Roche). Results shown are obtained from the same 3 organoids as used for Prestoblue analysis. Organoids in flasks were occasionally assayed to ensure consistency with the plates (data not shown). Prior to the assay start the dye solution and catalyst solution were mixed 45:1. One hundred microliters of test or control medium, $24 \mathrm{~h}$ following a complete media change, was assayed in a 96-well tissue culture plate in triplicate by adding $100 \mu \mathrm{l}$ of the dye-catalyst solution and incubating for 30 mins at room temperature. The reactions were terminated by addition of $50 \mu \mathrm{l}$ of stop solution. The plate was mixed in a ClarioSTAR plate reader (BMG) for $10 \mathrm{~s}$ before reading absorbance at $492 \mathrm{~nm}$.

\section{Immunofluorescence}

Organoids were fixed and immuno-stained as described previously [9]. FoxG1 (Abcam) and GFAP (Abcam) primary antibodies were used at a 1 in 50 dilution. Secondary Alexafluor 488 or 555 antibodies (Invitrogen) were applied at a 1 in 250 dilution and organoids were mounted in Fluoromount medium (ThermoFisher) with curing at room temperature for $24 \mathrm{~h}$.

\section{Histochemistry}

Antigen retrieval was performed as previously described [34], followed by staining using the anti-prion monoclonal antibody 6H4 (Prionics). Astrocyte detection was performed by staining with polyclonal rabbit anti-glial fibrillary acidic protein (anti-GFAP; Dako). Slides were also stained for observation of overall pathology using a standard hematoxylin-eosin (H\&E) protocol. All histopathology slides were analyzed by observers blinded to the inoculation groups using Aperio Imagescope software.

\section{RT-QulC}

Real-time QuIC (RT-QuIC) assays were performed similarly to those reported previously [30, 31]. Briefly, the RT-QuIC reaction mix contained $10 \mathrm{mM}$ phosphate buffer (pH 7.4), $300 \mathrm{mM} \mathrm{NaCl}, 0.1 \mathrm{mg} / \mathrm{ml}$ hamster recombinant
PrP 90-231 (purified as described in [31]), $10 \mu \mathrm{M}$ thioflavin $\mathrm{T}$ (ThT), and $1 \mathrm{mM}$ ethylenediaminetetraacetic acid tetrasodium salt (EDTA). Reaction mixes for culture media seeds (from the initial inoculum and samples collected throughout incubation) contained an additional $0.002 \%$ SDS in the reaction mix. Organoids were homogenized by motorized pestle to $10 \%(\mathrm{w} / \mathrm{v})$ in PBS and cleared with a $2000 \times g$ g min centrifugation. Organoid homogenates were serially diluted in $0.1 \% \mathrm{SDS} / \mathrm{PBS} / \mathrm{N} 2$ solution for a final SDS concentration of $0.002 \%$ in the reaction mix. For media seeded and organoid seeded reactions, respectively, either 80 or $98 \mu \mathrm{l}$ of reaction mix was loaded into a black 96-well plate with a clear bottom (Nunc), and reaction mixtures were seeded with $20 \mu \mathrm{l}$ of media or $2 \mu \mathrm{l}$ of the indicated dilution of organoid homogenate for a final reaction volume of $100 \mu \mathrm{l}$ and the same final concentrations in the reaction mix as indicated above. Plates were sealed (Nalgene Nunc International sealer) and incubated in a BMG FLUOstar Omega plate reader at $50{ }^{\circ} \mathrm{C}$ for 50 to $120 \mathrm{~h}$ with cycles of $60 \mathrm{~s}$ of shaking (700 rpm, double-orbital) and $60 \mathrm{~s}$ of rest throughout the incubation. ThT fluorescence measurements (excitation, $450 \pm 10 \mathrm{~nm}$; emission, $480 \pm 10 \mathrm{~nm}$ [bottom read]) were taken every $45 \mathrm{~min}$. Spearman-Kärber analyses [11] was used to provide estimates of the concentrations of seeding activity units giving positive reactions in $50 \%$ of replicate reactions, i.e., the $50 \%$ "seeding doses" or $\mathrm{SD}_{50}$ 's as previously described [42].

\section{Proteinase-K digests and Western blotting}

$10 \%$ organoid homogenates were treated with $5 \mu \mathrm{g} / \mathrm{ml}$ Proteinase $\mathrm{K}$ in $1 \%$ Sarkosyl for $1 \mathrm{~h}$ at $37^{\circ} \mathrm{C}$ with 400 rpm shaking. The reactions were stopped by incubation with $1 \mu \mathrm{M}$ Pefabloc for $5 \mathrm{~min}$ at $4{ }^{\circ} \mathrm{C}$. Samples were then mixed 1:1 with 2X Bolt LDS sample buffer (Invitrogen) containing $8 \% \beta$-mercaptoethanol and boiled for $10 \mathrm{~min}$. Samples were run on Bolt 4-12\% Bis-Tris gels (Invitrogen) and transferred to PVDF membranes using the iBlot 2 transfer system (Invitrogen). PrP was detected using the 3F4 antibody (Millipore) at a 1:10,000 dilution and visualized using ECL Select (Amersham) and imaged on the iBright imaging system (Invitrogen).

\section{Cytokine arrays}

A pool of conditioned media from flasks containing 6 organoids (media from the same 6 organoids was collected throughout) was collected at various time points during the incubation period. Media was assayed for cytokine levels using Bio-Plex Pro Human Inflammation Panel 1, 37-Plex (Biorad) as per the manufacturer's instructions. Cytokines below the level of detection throughout the experiment are excluded from the analysis. 


\section{Data analysis}

Positive pixel counts were carried out using ImageScope v11.1.2.760 imaging software (Aperio). Statistical analysis, as indicated in figure legends, was carried out in GraphPad Prism 7.04. Z-scores, heatmap, cluster analysis, and dendrogram of the cytokine protein secretion by cerebral organoids were produced using the online application Heatmapper (http://www2.heatmapper.ca/) developed in the Wishart Research Group at the University of Alberta [2]. The clustering method chosen was the Average Linkage setting, and the dendrogram distance measurement method chosen was the Pearson setting, which establishes the distance as the absolute value of the Pearson correlation coefficient (between 0 and 1).

\section{Results}

\section{Organoid infections}

Cerebral organoids develop mature astrocytic and oligodendrocytic populations from $\sim 2$ months, and these continue to increase in number to $\sim 5$ months with all organoids displaying some astrocytes by $\sim 140$ days [35]. Astrocytosis is prominent in prion diseases [22] and astrocytes have been shown to accumulate disease-associated
PrP prior to neuropathological changes [5, 10]. Therefore, we hypothesized that these cells may be important for the establishment of prion infection or the development of disease pathology. Human cerebral organoids were generated from induced pluripotent stem cells (iPSCs) [20]. To allow the cultures to develop glial populations, organoids were grown for 5 months (140 days) before commencing infections (organoid differentiation is shown in Fig. 1a with the timeline of infections in 1B). As previously mentioned, human prion disease phenotype and susceptibility are influenced by the methionine/valine polymorphism at amino acid position 129. The iPSCs used were heterozygous $(129 \mathrm{M} / \mathrm{V})$ for this polymorphism. To ensure optimal opportunity for uptake of infection by the organoids, we used brain homogenates from two SCJD patients who were also heterozygous at codon 129. In these sporadic cases, Proteinase-K resistant PrP had previously been glycotyped as type 1 or 2 CJD (nomenclature as described in [32]) and are referred to throughout as MV1 and MV2 accordingly. The MV1 and MV2 subtypes vary in disease duration and prevalence, with the MV1 subtype showing a generally faster disease progression but the MV2 subtype being $~ 3$ times more common than the MV1 [17, 33, 36]. Our

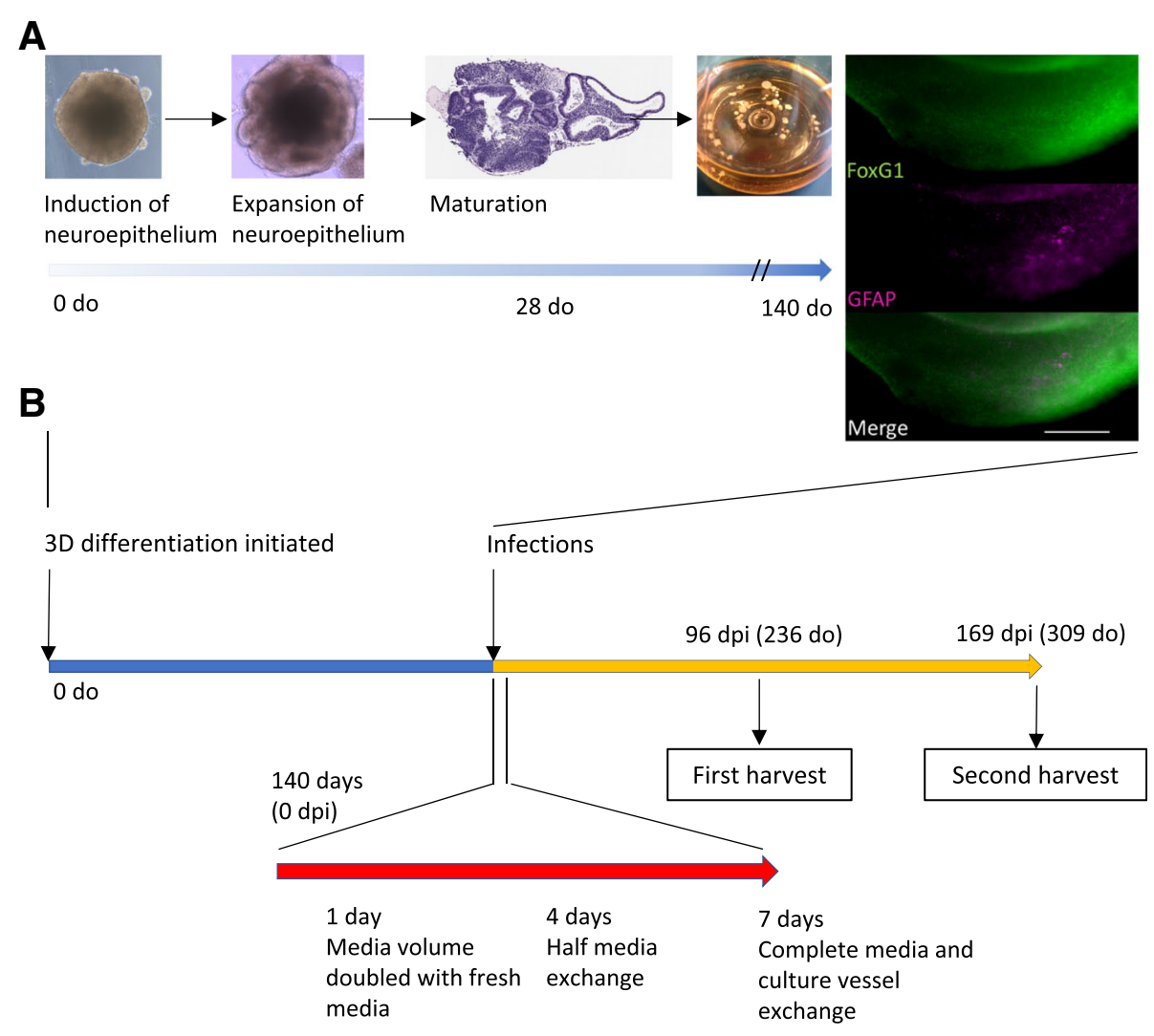

Fig. 1 Developmental and experimental schematic. a Example phase images show organoids at early stages of development, becoming more structured, and organoid appearance as balls of tissue in an Erlenmeyer culture flask. H\&E staining at 28 days old (do) shows the complexity of the organoids with many varied, structured domains. Immunofluorescence at 140 do confirms cortical identity (FoxG1) and shows that astrocytes (GFAP) have begun populating the neuronal layers. Scale bars $=200 \mu \mathrm{m}$. b Schematic time line of organoid maturation, infection and sampling 
inocula were from cases with a similar disease duration and age of onset (Table 1).

\section{Organoids exhibited inoculum-related changes in health} From the start of the infections, organoids were monitored for changes in their health using Prestoblue, a non-toxic probe that produces a fluorometric/colorimetric change within culture media in response to cell metabolism, including mitochondrial metabolism and enzymes involved in NADH/NADPH reactions. The MV1 inoculated organoids initially appeared to show slowing metabolism, but, 84 days post-infection (dpi), an almost two-fold increase was measured (Fig. 2a). This increase remained consistent for several weeks and then continued to climb until it fluctuated in the final weeks of the study. The MV2 inoculated organoids showed no change. At $92 \mathrm{dpi}$ increased LDH levels, indicative of cell lysis and death, were detected in the culture media of the MV1 inoculated organoids (but not the NBH or MV2; Fig. 2b). Therefore, three organoids per condition were harvested for IHC analysis at $96 \mathrm{dpi}$. The remaining organoids continued to be monitored by Prestoblue metabolism and periodic LDH. At 168 dpi the NBH exposed organoids showed an increase in LDH and a decrease in Prestoblue. This could have been indicative of the organoids starting to die from aging and, therefore, the remaining organoids were harvested for IHC or protein analysis at $169 \mathrm{dpi}$.

\section{Infected organoids were positive for RT-QulC seeding activity}

RT-QuIC analysis was used to monitor the organoids throughout the incubation period, by assaying culture media for prion seeding activity. One week after the full media exchange out of the inoculum at 7 days post addition (14 dpi), no RT-QuIC seeding activity remained within the media of the MV1 or MV2 organoids. For the $\mathrm{NBH}$ and MV1 inoculated organoids, culture media remained RT-QuIC negative for the duration of the study. However, seeding activity became detectable in the media of the organoids receiving the MV2 inoculum at 35 dpi (Fig. 3a).

Over the first few weeks of the infections, several organoids were harvested to determine the uptake of seeding activity from the inoculum and if, or how quickly, organoids could degrade the inoculum to an undetectable level. Organoids harvested at 7 days, the end of the inoculum exposure period, showed seeding activity likely resulting from remaining inoculum. MV1 organoids had less seeding activity uptake from the inocula than MV2, with only a low percentage of wells showing positivity per organoid (Fig. 3b). At 14 days, seeding was detected similarly but by $25-28$ dpi all inoculated organoids tested had cleared the original inoculum with no measurable seeding activity remaining in any well. For both inoculations seeding activity had re-appeared at $35 \mathrm{dpi}$. By comparison organoids harvested at the end of the experiment (169 dpi) showed 50\% or greater positive wells for the MV1 inoculated organoids and $100 \%$ positive for all MV2 organoids, indicating de novo production of seeding activity as opposed to persistence of the original inoculum within the tissue. Overall, the MV2 inoculated organoids showed greater seeding activity than the MV1 inoculated when harvested at $169 \mathrm{dpi}$ (Fig. 3c). MV2 inoculated organoids had an average seeding $\mathrm{SD}_{50}$ of $2.13( \pm 1.63) \times 10^{5}$ per $\mathrm{mg}$ of tissue, while the MV1 inoculated organoids had an average $\mathrm{SD}_{50}$ of $1.69( \pm 0.70) \times 10^{3}$ per $\mathrm{mg}$ of tissue (Fig. 3d) and $\mathrm{NBH}$ treated organoids showed no seeding activity (Fig. 3c).

\section{MV2 infected organoids showed protease-resistant PrP}

Protease-resistant PrP is often considered a hallmark of prion disease. Western blotting, following proteinase-K digestion, of the organoid tissue harvested at $169 \mathrm{dpi}$ showed clear protease-resistant PrP banding in all of
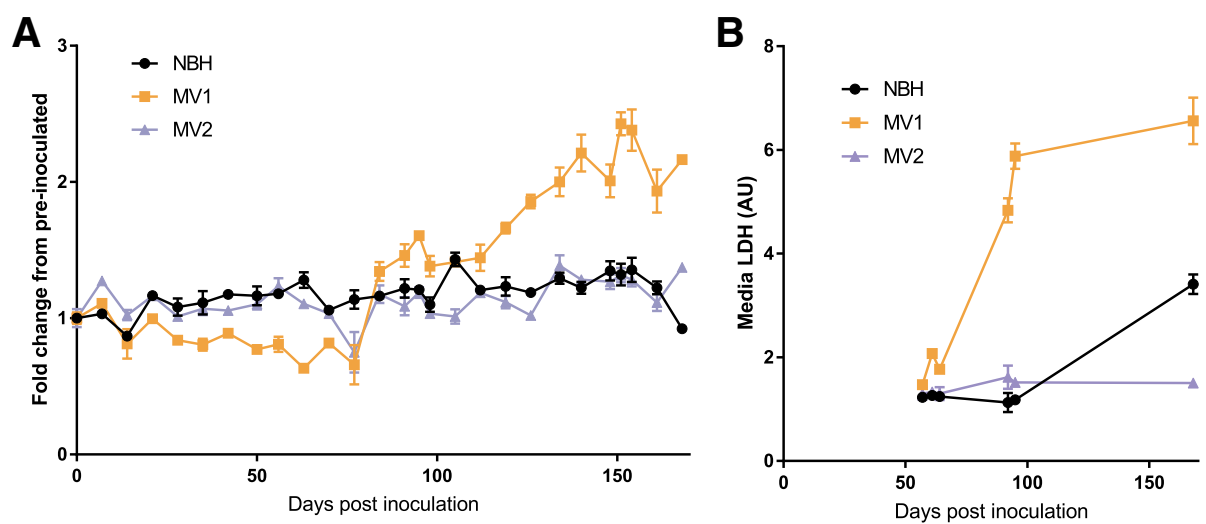

Fig. 2 Organoid health and viability monitoring. a Prestoblue fluorescence, indicating changes in metabolism, expressed relative to preinoculated organoid metabolism. The same three organoids were monitored weekly throughout. $\mathbf{b}$ LDH detection in organoid culture media, indicative of cell death or loss of membrane integrity, of the same 3 organoids as in A. Data points shown mean and SD of 3 replicate reads 
A Conditioned media

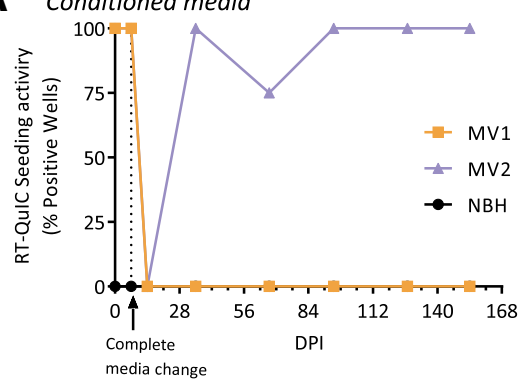

C Organoids $169 \mathrm{dpi}$

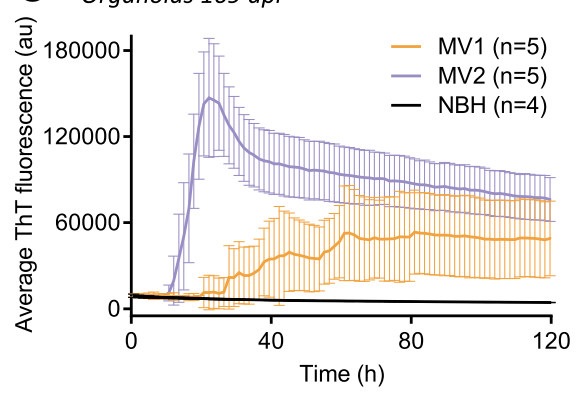

B Organoids

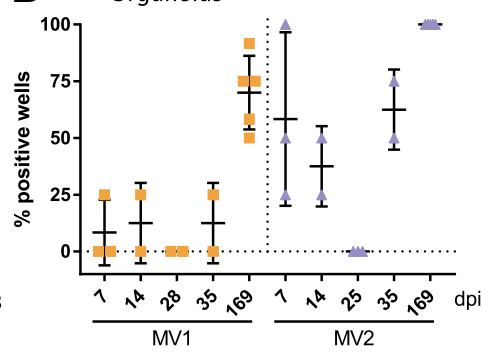

D Organoids 169 dpi

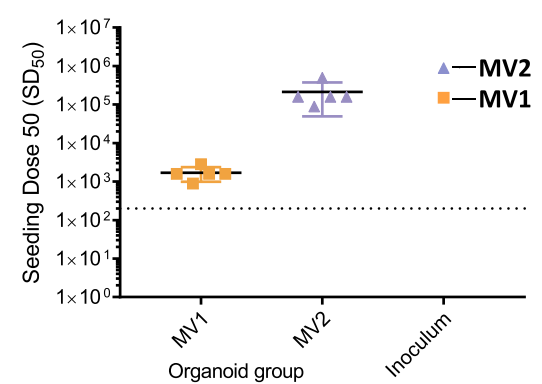

E

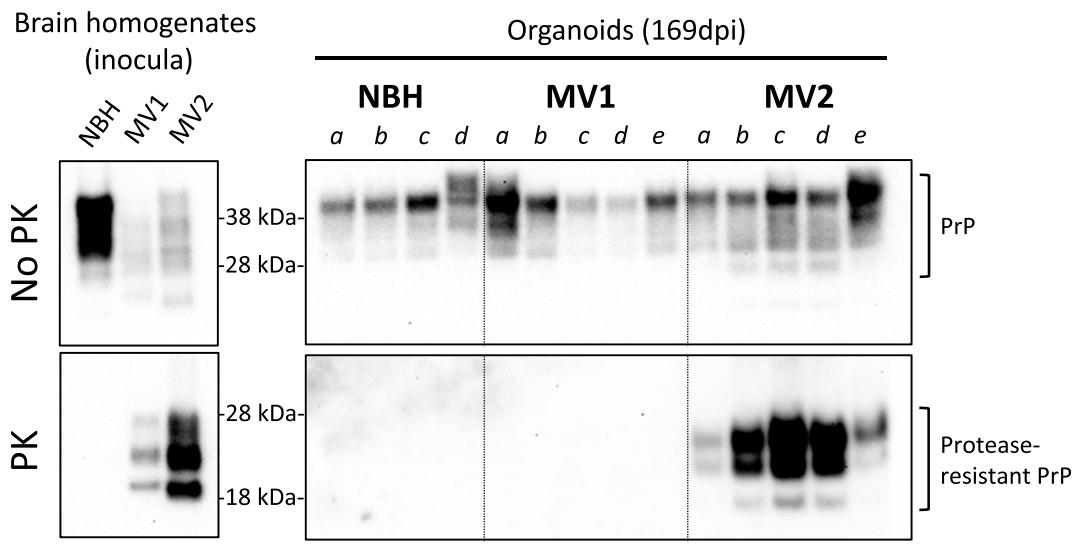

Fig. 3 RT-QulC seeding and protease resistant PrP analysis. a Media RT-QulC monitoring throughout the duration of infection showing the number of replicate wells positive for seeding activity at each sampling time. Each data point represents media taken from the well containing the same three organoids in Fig. 2. Minor tick marks on the x-axis show weekly full media exchanges. $\mathbf{b}$ Percentage of positive RT-QulC test wells of organoids taken at various early time points during infection and when harvested at the conclusion on the experiment (169dpi). Each data point represents an individual organoid tested in RT-QulC with 4-12 replicate wells. Bars show mean and standard deviation of all organoids in each indicated group. c RT-QulC seeding activity of organoid homogenates diluted $10^{-3}$ following harvest at 169 dpi. For clarity, every other datapoint was plotted. RT-QulC traces are displayed as averages from all organoids in each group. Error bars represent standard deviation. $\mathbf{d} \mathrm{SD}_{50}$ values of each organoid harvested at $169 \mathrm{dpi}$ and the original inoculums. Each point represents an individual organoid (or inoculum) with black lines denoting the mean and colored bars the standard deviation. The dotted line represents the threshold of detection below which results are considered negative. e Western blots showing PrP and protease-resistant PrP in the original brain homogenate inocula (left plate) and the organoids harvested at 169 dpi (right plate)

the MV2 treated organoids with an apparent shift towards a di-glycosylation dominant banding pattern with less unglycosylated PrP (Fig. 3e). Protease-resistant PrP was undetectable by western blot in the MV1 treated organoids, consistent with the lower RT-QuIC seeding activity. $\mathrm{PrP}^{\mathrm{Res}}$ could not be detected the MV1 infected organoids by western blot even following sodium phosphotungstic acid precipitation (data not shown).

\section{PrP immunostaining was altered in MV2 inoculated organoids}

During CJD, prions accumulate within the brain resulting in different immunohistochemical appearances of $\operatorname{PrP}$ from the normal protein found in individuals without prion disease. In the cerebral organoids, total PrP immunohistochemical staining was not significantly increased as a result of infection at either $96 \mathrm{dpi}$ (Additional file 1: Figure S1) or 169 dpi (Fig. 4a/b). PrP staining was often 

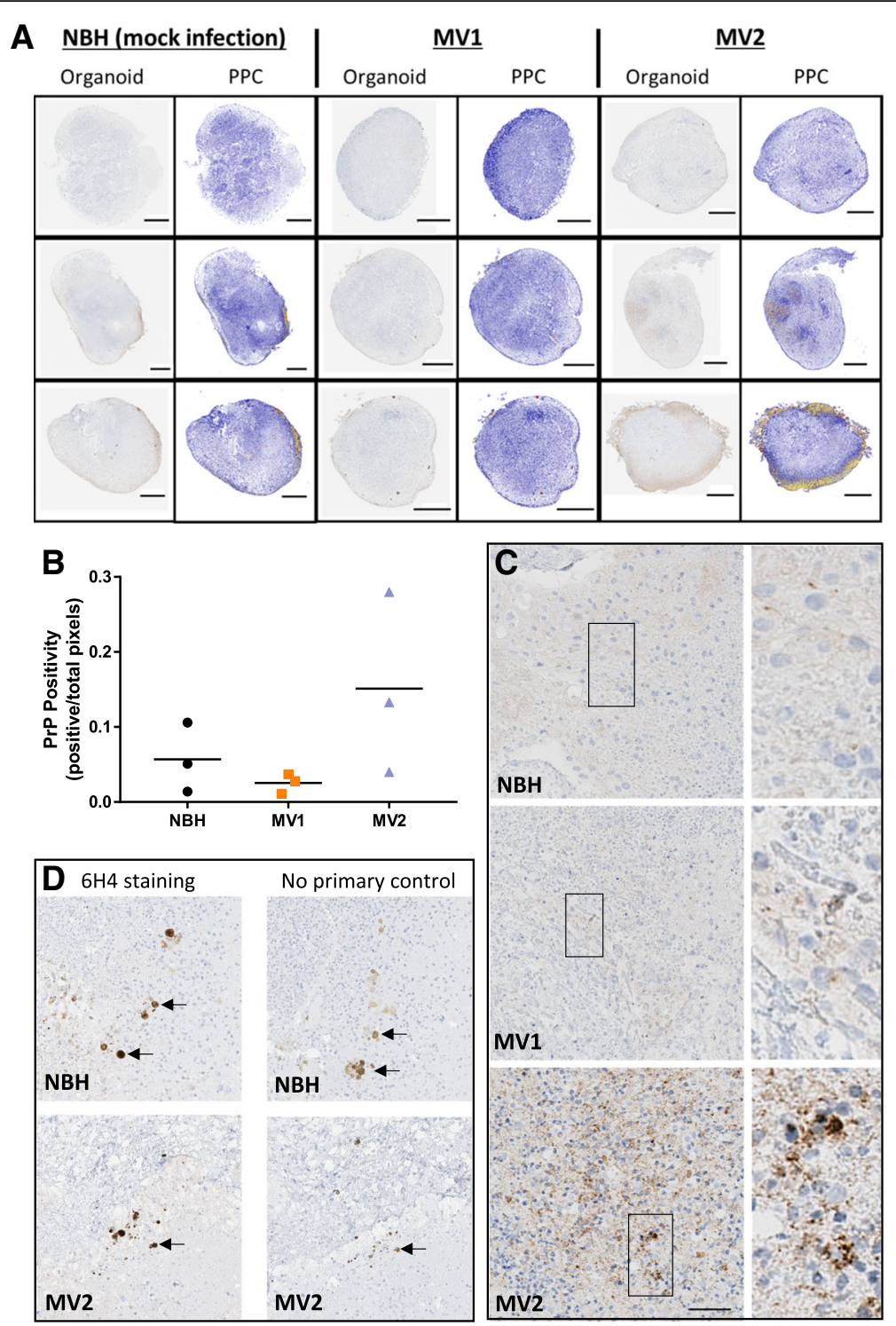

Fig. $4 \mathrm{Imm}$ unohistochemical staining for PrP in organoids harvested at $169 \mathrm{dpi}$. a Whole organoid images; left panels per condition show $6 \mathrm{H} 4$ PrP staining (brown) and right panels showing positive pixel counts (PPC), blue signifies low and yellow-orange denotes high pixel staining intensity. Scale bars $=500 \mu \mathrm{m}$. b PrP positive pixel counts. Each data point represents an individual organoid with the mean indicated as a line (Kruskal-Wallis, $p=0.132$ ). c $6 \mathrm{H} 4$ PrP staining of organoid interiors. Scale bar $=50 \mu \mathrm{m}$ and applies to left panels. Right panels are zoomed images of the boxed regions. $\mathbf{d}$ PrP staining artifacts visible in NBH and MV2 treated organoids. Left panels show 6H4 PrP staining and right panels show no-primary antibody control stains. Arrows indicate artifacts in sequential slices. Scale is as for C

seen toward the periphery of the organoid regardless of inoculum (Fig. 4a). However the MV2 organoids showed areas of PrP staining internally. The internal staining of the MV2 inoculated organoids showed areas of course, punctate, granular PrP indicative of a sporadic CJD-like staining pattern, while the MV1 organoids were indistinguishable from controls (Fig. 4c). Some artifactual staining was additionally observed in both the controls and the MV2 stained organoids (Fig. 4d); this was unrelated to pathology as it was visible in the sequential no-primary antibody control sections. These pigments are often found within healthy organoids, visible by eye when observing organoids in culture, and may be retinal pigment or neuromelanin [20]. In the case of the latter, an enhanced pigment intensity due to $\operatorname{PrP}$ immunoreactivity would not be unexpected as PrP has been shown to bind to melanin pigments [15].

\section{Histochemistry showed no differences between SCJD inoculated and control organoids}

Vacuolation and astrogliosis are further hallmarks of prion disease. Therefore, this was assessed by hematoxylin and 
eosin (H\&E) histochemistry. Organoids showed diverse regions of organized cells with different staining intensities, consistent with previous descriptions of organoid self-organization [20, 35](Fig. 5a). At 96 dpi, vacuolation was apparent in all three organoids given the MV1 inocula and in two of the three MV2 treated organoids but also in one of three of the NBH treated organoids, indicating that vacuolation occurs from non-prion causes as a normal event within these tissues. At $169 \mathrm{dpi}$, the NBH treated organoids showed significant vacuolation (Fig. $5 \mathrm{~b}$ ) indicating that this parameter cannot be used as a measure of disease pathology in these organoids.

Astrogliosis was assessed by detection of glial fibrillary acidic protein (GFAP), an astrocyte marker. At 96 dpi, GFAP staining appeared increased in both the MV1 and MV2 inoculated organoids, however one of the control
$\mathrm{NBH}$ organoids also had a high density of astrocytes indicating this may not be disease specific but reflect the rate of maturation within the heterogenous organoids (Fig. 6a and b). GFAP staining was highly increased 169 dpi in all organoids including controls (Additional file 1: Figure S2). As GFAP expression is known to increase in normal astrocytes during aging [7, 28], this traditional marker of astrogliosis in prion disease may have limited utility for organoid studies where cultures are aged for long time periods.

An alternative measure of astrocyte activation is variation in cytokine secretion indicative of neuroinflammation. Neuroinflammation is usually mediated by microglia and astrocytes, but, since the organoids lack microglia (microglia differentiate from mesoderm rather than the induced neuroectoderm), changes detected can be

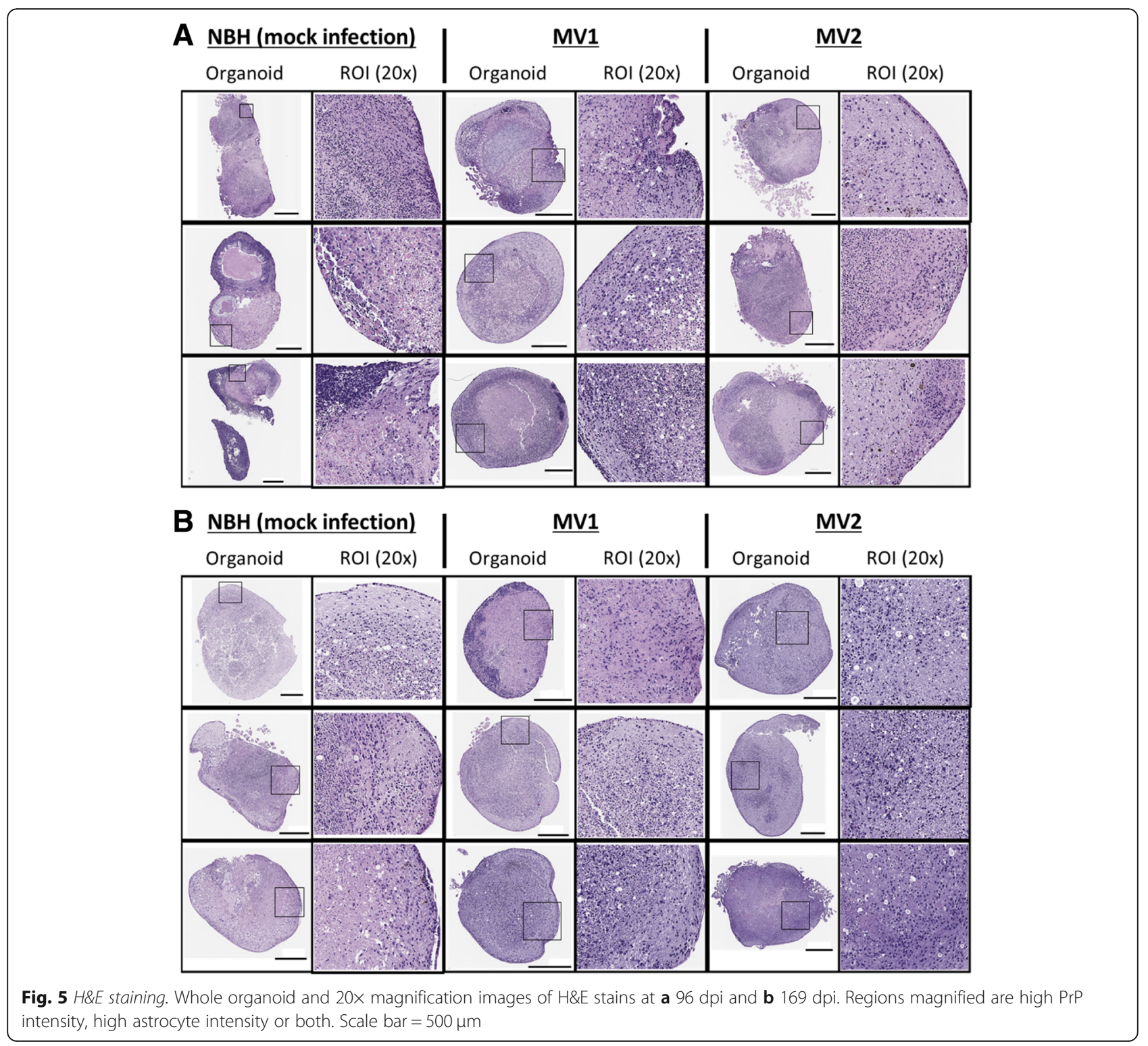



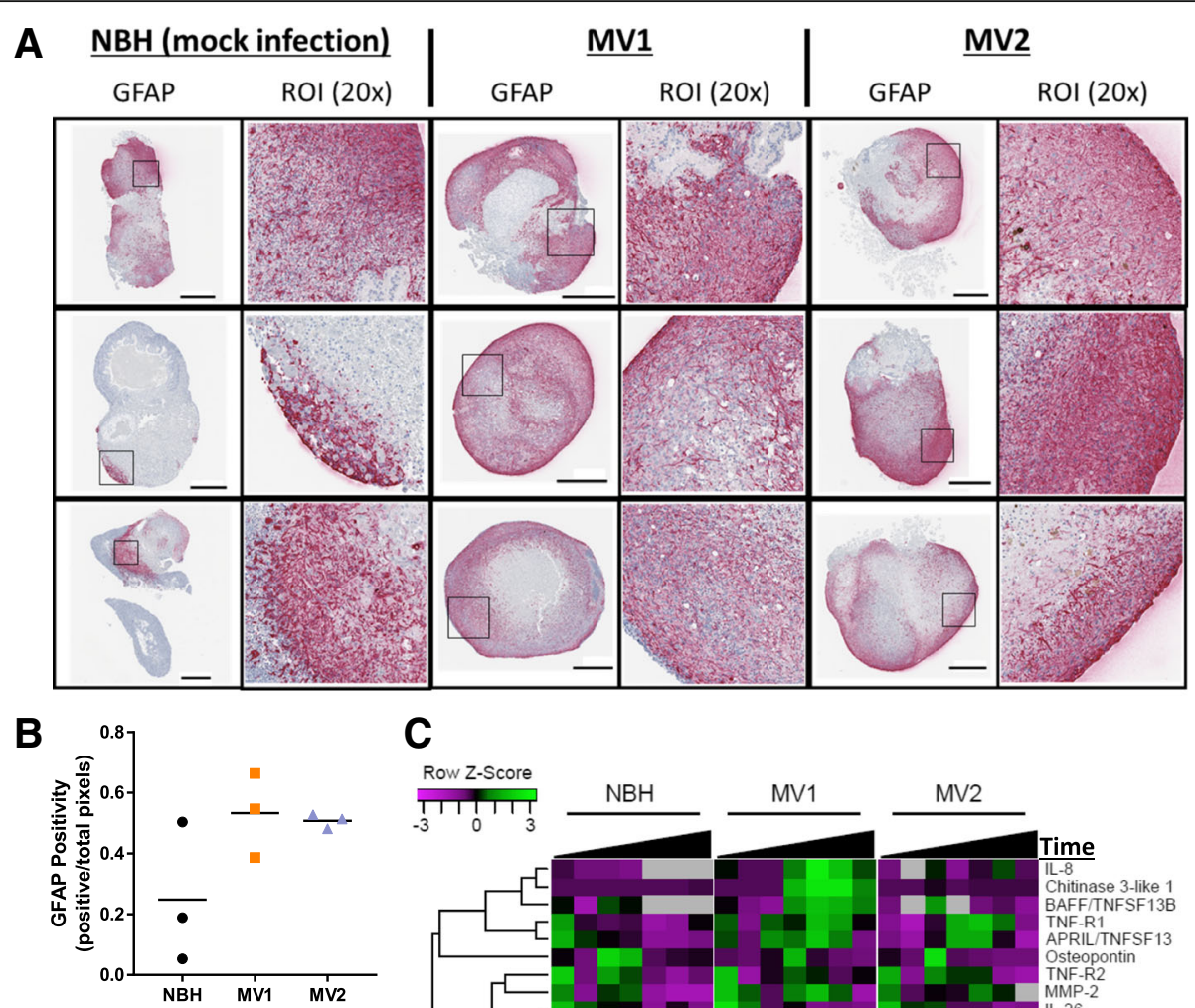

Cow z-Score
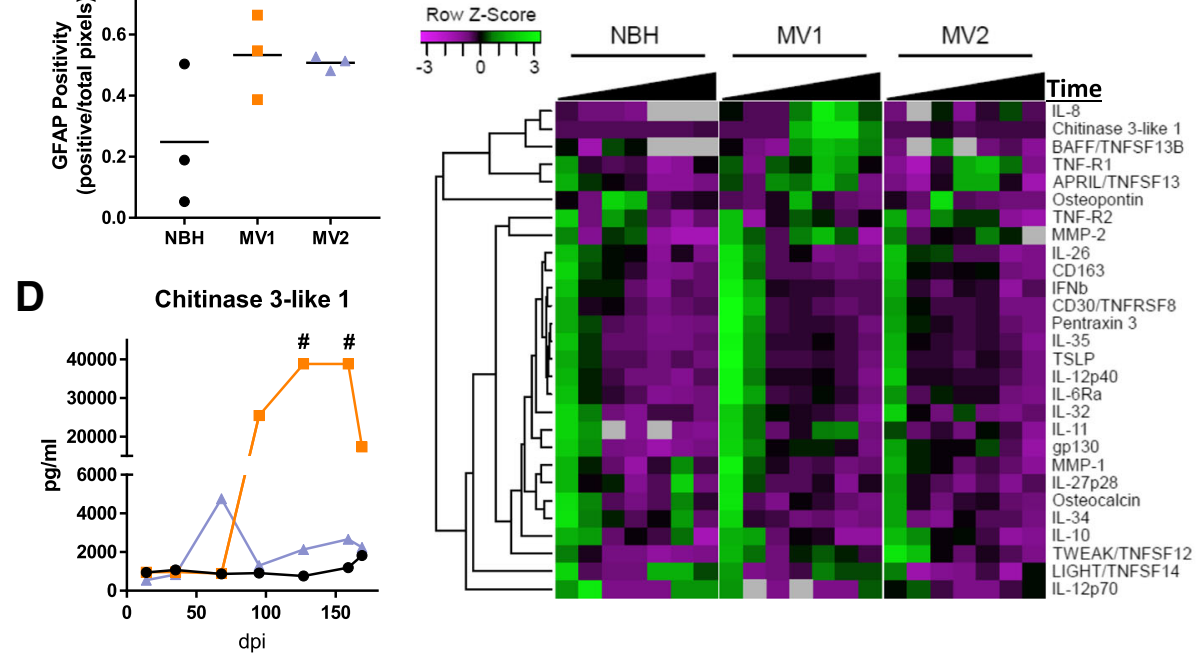

Fig. 6 Astrocyte changes during infection. a Whole organoid images at 96 dpi; left panels per condition show GFAP staining (pink) and right panels showing positive pixel counts (PPC), blue signifies low and yellow-orange denotes high pixel staining intensity. Scale bars $=500 \mu \mathrm{m}$. $\mathbf{b}$ GFAP positive pixel counts of the 96 dpi whole organoid images. Each data point represents an individual organoid with the mean indicated as a line (Kruskal-Wallis, $p=0.196)$. c Heatmap and dendrogram cluster analysis of cytokine detection in culture medium at 14, 35, 68, 95, 127, 159, 169 dpi. Black triangles showing increasing time from inoculation. Data points are generated using media pools from 6 individual organoids. Grey boxes indicate cytokine concentrations below the limits of detection. $\mathbf{d}$ Concentrations of Chitinase 3 -like 1 in culture medium over time. \# readings exceeded the accurate range of detection for the assay

attributed primarily to astrocytes. We should note here that while a recent study has shown microglia can differentiate within cerebral organoid cultures from residual mesoderm-derived progenitors [29], no Iba1 positive cell staining (indicative of microglia) was observed in our organoid cultures (data not shown). Whilst microglia are thought to play an important role in prion disease pathogenesis, recent research has shown that they are not necessary for disease progression, appearing to play a protective role against pathogenesis [6], and so we deemed their absence would not hinder disease manifestation within the organoids. For cytokine screening, media pools were collected at 14 , $35,68,95,127,159$, and 169 dpi and cluster analysis was used to group cytokines such that the most closely correlated detection patterns are aligned. Most detected cytokines were highest at $14 \mathrm{dpi}$ and then declined in all organoids regardless of inoculum (Fig. 6c). However, a cluster of cytokines with increased expression from $95 \mathrm{dpi}$ was observed in the culture medium from the MV1 inoculated organoids, including IL-8, chitinase 3-like 1, BAFF/ TNFSF13B, TNFR1 and APRIL/TNFSF13. The MV2 inoculated organoids showed a similar increase in TNF-R1 and APRIL/TNFSF13 but otherwise showed little change 
from NBH inoculated organoids. Notably, the increase in chitinase 3-like 1 secretion measured for the MV1 inoculated organoids was almost 30 -fold, with two samplings (at 127 and 159 dpi) showing readings in excess of the accurate limit of detection.

\section{Discussion}

Herein, we have demonstrated that iPSC-derived human cerebral organoid cultures can be used to model prion transmission, propagation and limited disease pathology. We show that two different sporadic CJD inocula affect the same underlying organoid line differently, supporting that the prion seed greatly influences the disease phenotype in genetically identical human cells. Our data also indicated that the underlying cell type itself influences the protease-resistant prions that are formed; in all of the MV2 organoids, a shift from the monoglycosylation dominant banding pattern of the SCJD inoculum to diglycosylation dominant indicates that the prions have adapted as they propagated within the organoids.

The MV2 inoculum produced more overt measures of infection in the organoids than the MV1 inoculum, with clear protease-resistant $\operatorname{PrP}$ and PrP tissue deposition. The MV1 inoculated organoids generated de novo seeding activity but did not show these traditional hallmarks of prion infection. It is probable that the MV1 PrP seeding the RT-QuIC reaction was close to the limits of detection, and consequently too low to be detected by traditional techniques. Alternatively, the newly synthesized MV1 prions might be protease sensitive. The presence of protease sensitive $\operatorname{PrP}$ is a possibility since as much as $90 \%$ of the mis-folded PrP in CJD brain tissue has been reported to be protease sensitive [37], and in mouse models of prion infection, $\operatorname{PrP}$ mutations that cause accumulation of protease sensitive prions result in a highly neurotoxic species [8]. Preliminary consideration of insoluble $\operatorname{PrP}$ within the organoids indicated that it may be present in some MV1 infected organoids (data not shown), therefore the role of protease sensitive $\operatorname{PrP}$ warrants further investigation in future studies. Despite the lack of hallmark indicators of PrP infection, the MV1 inoculum demonstrated more influence on health parameters. We can speculate that this could be a property of the MV1 prions; potentially, a more neurotoxic phenotype might correlate with the shorter median clinical course often observed in MV1 sCJD, typically 4-5 months as compared with $\sim 11-17$ months in MV2 sCJD $[17,33,36]$. However, whether this is related to subtype or an inoculum-specific effect will not be answerable until a greater number of inocula have been screened within this system.

Other factors may have influenced the differences observed between the two inoculas. Prions spread from cell to cell by a number of methods including tunneling nanotubules and exosomal release [12, 41, 47]. Using mouse prion strains it has been shown that the amount of infectivity released through the exosomal pathway is highly strain dependent [1], and this may also be true for the sCJD subtypes. The prions released into the media through the exosomal pathway may be much lower for the MV1 inoculated organoids than the MV2, resulting in a lack of detection within the media and slower propagation of prions through the tissue. Secretion of the MV2 prions into the culture medium may also have aided spread between the organoids in each culture vessel. The lack of detectable seeding activity from the media of the MV1 inoculated organoids is most likely due to released prions being below the threshold of detection.

Cellular heterogeneity may also contribute to the difference between the organoids inoculated with the MV1 and MV2 prions. During the formation and development of the organoids, various cell populations are patterned [43]. Prion subtypes often exhibit different histopathological lesions within different brain regions. The heterogeneity of the organoids may result in a preference for uptake of an inoculum that attacks the specific neuronal populations developed within the organoid. MV1 infection levels may be lower as organoids contain less neurons capable of propagating MV1 prions or because MV1 prions are more toxic to these populations, causing their death before they have propagated new prions. Organoid technology is continuing to progress, such that organoids can be stimulated to differentiate in specific directions, such as dorsal or ventral forebrain and cerebellum $[27,40]$. This utility could prove highly valuable in understanding why different prion subtypes preferentially attack specific brain regions. A further exciting development in organoid technologies is the merging of organoids composed of different brain regions and the exchange of cells that results $[3,43]$. This could be adapted to provide valuable information on the spreading of prions from cell to cell within the human brain.

While the MV1 uptake and propagation was clearly lower than observed for the MV2, the MV1 infected organoids showed changes in health as a result of exposure to the inoculum, including increased metabolism, LDH release and cytokine release. Chitinase 3-like 1, Chi3L1 or YKL-40, is an inflammatory cytokine primarily produced in adult astrocytes [46], which can induce death of neurons and oligodendrocytes $[23,45]$. Chi3L1 has previously been reported to be increased in MM1 and VV2 sCJD brain [24]. MV1 and MM1 sCJD are grouped as the same subtype due to the similarities in pathology of these two diseases $[13,32]$, therefore the increase in this cytokine in the MV1 inoculated organoids is consistent with expected in vivo changes. The lack of Chi3L1 change in the MV2 organoids is unclear but detection may have increased had these organoids been cultured for longer. 
Infection of hu-iPSC derived neuronal cultures has been attempted previously using both 'normal' cells and cells carrying the prion Y218N mutation [26]. These infections were unsuccessful, but there may be several reasons that explain the difference from our own study. First, the hu-iPSCs infected in the Y218N study were differentiated as monolayers, which may lose infected cells over serial passages. Second, the authors followed the cultures until 77-79 dpi, but our model had not shown PrP deposition in the tissues at $96 \mathrm{dpi}$. Therefore, longer incubations may be required to demonstrate infection, which may or may not be possible in the previous model.

Our model demonstrates that cerebral organoids can propagate prion infection from SCJD sources, and that different sCJD subtypes may manifest differently within the same underlying cell. Whilst this latter point is currently being validated, requiring a greater number of inocula to be screened beyond the two tested herein, the capability of organoids differentiated from the same underlying iPSCs to differentiate distinct sCJD subtypes based on their biological properties may be of immense value for understanding the pathologies caused by different human prions.

\section{Conclusion}

Human cerebral organoids are a promising new system for modelling prion disease in cell culture. Organoids take up prion infection, influenced by the prion seed to which they are exposed, and generate new seeding activity. Furthermore, they can produce glycosylated protease-resistant $\operatorname{PrP}$ and $\operatorname{PrP}$ deposition. We acknowledge that the cerebral organoids have clear limitations; they are highly heterogenous, not vascularized and they may lack non-neuronally derived cells of the brain such as epithelial cells and microglia. Despite these limitations, these cultures represent the closest in vitro cell model to human brain currently available and offer the first human three-dimensional model of prion disease. Their use holds promise for the investigation of different subtype pathologies, for investigation of how prions spread throughout the brain and for trialing putative therapeutics in a human tissue background.

\section{Additional file}

Additional file 1: Figure S1. Whole organoid PrP staining at 96dpi. Upper panels display whole organoid images; left panels per condition show $6 \mathrm{H} 4 \mathrm{PrP}$ staining (brown) and right panels showing positive pixel counts (PPC), blue signifies low and yellow-orange denotes high pixel staining intensity. Scale bars $=500 \mu \mathrm{m}$. Lower graph shows PrP positive pixel counts. Each data point represents an individual organoid with the mean indicated as a line. Figure S2. GFAP staining of organoids. A. Panels display whole organoid (left) and 20x magnified images of regions of interest (right) of organoids harvested at $169 \mathrm{dpi}$. B. Positive pixel counts (PPC) at 96 and 169 dpi, blue signifies low and yellow-organe denotes high pixel staining intensity. Scale bars $=500 \mu \mathrm{m}$. C. GFAP positive pixel count quantification at $169 \mathrm{dpi}$. Each data point represents an indevidual organoid with the mean indicated as a line. (DOCX $1790 \mathrm{~kb}$ )

\section{Acknowledgements}

The authors would like to thank Nancy Kurtz and Lori Lubke for technical immunohistochemistry assistance, Lynne Raymond for codon 129 sequencing expertise, Andrew Hughson for preparation of recombinant prion protein, and Bruce Chesebro, Byron Caughey, and Sonja Best for critical evaluation of this manuscript.

\section{Funding}

The preliminary funding to begin this work was gratefully received from a Silva Coelho memorial grant (administered by the CJD support group network, Australia). This research was supported by the Intramural Research Program of the NIH (NIAID).

\section{Availability of data and materials}

The datasets during and/or analysed during the current study are available from the corresponding author on reasonable request.

\section{Authors' contributions}

All authors contributed experimental materials, data and analysis. BRG and $\mathrm{CLH}$ wrote the manuscript. SF, CDO, GZ, JAC, BR edited and approved the final submission. All authors read and approved the final manuscript.

Ethics approval and consent to participate

Not applicable.

Consent for publication

Not applicable.

Competing interests

The authors declare that they have no competing interests.

\section{Publisher's Note}

Springer Nature remains neutral with regard to jurisdictional claims in published maps and institutional affiliations.

\section{Author details}

${ }^{1}$ Division of Intramural Research, Laboratory of Persistent Viral Diseases, National Institute of Allergy and Infectious Diseases, Rocky Mountain Laboratories, National Institutes of Health, 903 South 4th Street, Hamilton, MT 59840, USA. ²Department of Neurosciences, Biomedicine and Movement Sciences, University of Verona, Verona, Italy.

Received: 29 March 2019 Accepted: 16 May 2019

Published online: 14 June 2019

\section{References}

1. Arellano-Anaya ZE, Huor A, Leblanc P, Lehmann S, Provansal M, Raposo G, Andreoletti O, Vilette D (2015) Prion strains are differentially released through the exosomal pathway. Cellular and molecular life sciences. CMLS 72:1185-1196. https://doi.org/10.1007/s00018-014-1735-8

2. Babicki S, Arndt D, Marcu A, Liang Y, Grant JR, Maciejewski A, Wishart DS (2016) Heatmapper: web-enabled heat mapping for all. Nucleic Acids Res 44:W147-W153. https://doi.org/10.1093/nar/gkw419

3. Bagley JA, Reumann D, Bian S, Levi-Strauss J, Knoblich JA (2017) Fused cerebral organoids model interactions between brain regions. Nat Methods 14:743-751. https://doi.org/10.1038/nmeth.4304

4. Cali I, Castellani R, Yuan J, Al-Shekhlee A, Cohen ML, Xiao X, Moleres FJ, Parchi P, Zou WQ, Gambetti P (2006) Classification of sporadic Creutzfeldt-Jakob disease revisited. Brain 129:2266-2277. https://doi.org/10.1093/brain/awl224

5. Carroll JA, Chesebro B (2019) Neuroinflammation, microglia, and cellassociation during prion disease. Viruses 11. https://doi.org/10.3390/v11010065

6. Carroll JA, Race B, Williams K, Striebel J, Chesebro B (2018) Microglia are critical in host defense against prion disease. J Virol. https://doi.org/10.1128/jvi.00549-18

7. Clarke LE, Liddelow SA, Chakraborty C, Munch AE, Heiman M, Barres BA (2018) Normal aging induces A1-like astrocyte reactivity. Proc Natl Acad Sci U S A 115:E1896-e1905. https://doi.org/10.1073/pnas.1800165115

8. Coleman BM, Harrison CF, Guo B, Masters CL, Barnham KJ, Lawson VA, Hill AF (2014) Pathogenic mutations within the hydrophobic domain of the prion protein lead to the formation of protease-sensitive prion species with increased lethality. J Virol 88:2690-2703. https://doi.org/10.1128/jvi.02720-13 
9. Collins SJ, Haigh CL (2017) Simplified murine 3D neuronal cultures for investigating neuronal activity and neurodegeneration. Cell Biochem Biophys 75:3-13. https://doi.org/10.1007/s12013-016-0768-z

10. Diedrich JF, Bendheim PE, Kim YS, Carp Rl, Haase AT (1991) Scrapieassociated prion protein accumulates in astrocytes during scrapie infection. Proc Natl Acad Sci U S A 88:375-379

11. Dougherty RM (1964) Animal virus titration techniques. In: Harris RJC (ed) Techniques in experimental virology. Academic, New York, pp 183-186

12. Fevrier B, Vilette D, Archer F, Loew D, Faigle W, Vidal M, Laude H, Raposo G (2004) Cells release prions in association with exosomes. Proc Natl Acad Sci U S A 101:9683-9688. https://doi.org/10.1073/pnas.0308413101

13. Gambetti P, Kong Q, Zou W, Parchi P, Chen SG (2003) Sporadic and familial CJD: classification and characterisation. Br Med Bull 66:213-239

14. Gonzalez C, Armijo E, Bravo-Alegria J, Becerra-Calixto A, Mays CE, Soto C (2018) Modeling amyloid beta and tau pathology in human cerebral organoids. Mol Psychiatry. https://doi.org/10.1038/s41380-018-0229-8

15. Hamanaka T, Nishizawa K, Sakasegawa Y, Oguma A, Teruya K, Kurahashi H, Hara H, Sakaguchi S, Doh-Ura K (2017) Melanin or a melanin-like substance interacts with the $\mathrm{N}$-terminal portion of prion protein and inhibits abnormal prion protein formation in prion-infected cells. J Virol 91. https://doi.org/10.1128/vivi.01862-16

16. Hill AF, Joiner S, Wadsworth JD, Sidle KC, Bell JE, Budka H, Ironside JW Collinge J (2003) Molecular classification of sporadic Creutzfeldt-Jakob disease. Brain 126:1333-1346

17. Knight R (2017) Infectious and sporadic prion diseases. Prog Mol Biol Transl Sci 150:293-318. https://doi.org/10.1016/bs.pmbts.2017.06.010

18. Krejciova Z, Alibhai J, Zhao C, Krencik R, Rzechorzek NM, Ullian EM, Manson J, Ironside JW, Head MW, Chandran S (2017) Human stem cell-derived astrocytes replicate human prions in a PRNP genotype-dependent manner. J Exp Med 214:3481-3495. https://doi.org/10.1084/jem.20161547

19. Ladogana A, Liu Q, Geng Xi Y, Pocchiari M (1995) Proteinase-resistant protein in human neuroblastoma cells infected with brain material from Creutzfeldt-Jakob patient. Lancet 345:594-595. https://doi.org/10.5555/uri. S0140673695905081

20. Lancaster MA, Knoblich JA (2014) Generation of cerebral organoids from human pluripotent stem cells. Nat Protoc 9:2329-2340. https://doi.org/10. 1038/nprot.2014.158

21. Lancaster MA, Renner M, Martin CA, Wenzel D, Bicknell LS, Hurles ME, Homfray T, Penninger JM, Jackson AP, Knoblich JA (2013) Cerebral organoids model human brain development and microcephaly. Nature 501: 373-379. https://doi.org/10.1038/nature12517

22. Liberski PP, Brown P (2004) Astrocytes in transmissible spongiform encephalopathies (prion diseases). Folia Neuropathol 42(Suppl B):71-88

23. Liddelow SA, Guttenplan KA, Clarke LE, Bennett FC, Bohlen CJ, Schirmer L, Bennett ML, Munch AE, Chung WS, Peterson TC et al (2017) Neurotoxic reactive astrocytes are induced by activated microglia. Nature 541:481-487. https://doi.org/10.1038/nature21029

24. Llorens F, Thune K, Tahir W, Kanata E, Diaz-Lucena D, Xanthopoulos K, Kovatsi E, Pleschka C, Garcia-Esparcia P, Schmitz M et al (2017) YKL-40 in the brain and cerebrospinal fluid of neurodegenerative dementias. In: Molecular neurodegeneration, vol 12, p 83. https://doi.org/10.1186/s13024-017-0226-4

25. Marton RM, Miura Y, Sloan SA, Li Q, Revah O, Levy RJ, Huguenard JR, Pasca SP (2019) Differentiation and maturation of oligodendrocytes in human three-dimensional neural cultures. Nat Neurosci. https://doi.org/10.1038/ s41593-018-0316-9

26. Matamoros-Angles A, Gayosso LM, Richaud-Patin Y, di Domenico A, Vergara C, Hervera A, Sousa A, Fernandez-Borges N, Consiglio A, Gavin R et al (2018) iPS cell cultures from a Gerstmann-Straussler-Scheinker patient with the Y218N PRNP mutation recapitulate tau pathology. Mol Neurobiol 55:30333048. https://doi.org/10.1007/s12035-017-0506-6

27. Muguruma K (2018) Self-organized cerebellar tissue from human pluripotent stem cells and disease modeling with patient-derived iPSCs. Cerebellum 17: 37-41. https://doi.org/10.1007/s12311-017-0905-2

28. Nichols NR, Day JR, Laping NJ, Johnson SA, Finch CE (1993) GFAP mRNA increases with age in rat and human brain. Neurobiol Aging 14:421-429

29. Ormel PR, Vieira de Sa R, van Bodegraven EJ, Karst H, Harschnitz O, Sneeboer MAM, Johansen LE, van Dijk RE, Scheefhals N Berdenis van Berlekom Aet al (2018) microglia innately develop within cerebral organoids. Nat Commun 9:4167. https://doi.org/10.1038/s41467-018-06684-2

30. Orru CD, Groveman BR, Hughson AG, Zanusso G, Coulthart MB, Caughey B (2015) Rapid and sensitive RT-QulC detection of human Creutzeldt-Jakob disease using cerebrospinal fluid. MBio 6. https://doi.org/10.1128/mBio.02451-14
31. Orru CD, Hughson AG, Groveman BR, Campbell KJ, Anson KJ, Manca M, Kraus A, Caughey B (2016) Factors that improve RT-QulC detection of prion seeding activity. Viruses 8. https://doi.org/10.3390/v8050140

32. Parchi P, de Boni L, Saverioni D, Cohen ML, Ferrer I, Gambetti P, Gelpi E, Giaccone G, Hauw JJ, Hoftberger R et al (2012) Consensus classification of human prion disease histotypes allows reliable identification of molecular subtypes: an inter-rater study among surveillance centres in Europe and USA. Acta Neuropathol 124:517-529. https://doi.org/10.1007/s00401-012-1002-8

33. Parchi P, Giese A, Capellari S, Brown P, Schulz-Schaeffer W, Windl O, Zerr I, Budka H, Kopp N, Piccardo P et al (1999) Classification of sporadic Creutzfeldt-Jakob disease based on molecular and phenotypic analysis of 300 subjects. Ann Neurol 46:224-233

34. Race B, Williams K, Orru CD, Hughson AG, Lubke L, Chesebro B (2018) Lack of transmission of chronic wasting disease to Cynomolgus macaques. J Virol. https://doi.org/10.1128/JVI.00550-18

35. Renner M, Lancaster MA, Bian S, Choi H, Ku T, Peer A, Chung K, Knoblich JA (2017) Self-organized developmental patterning and differentiation in cerebral organoids. EMBO J 36:1316-1329. https://doi. org/10.15252/embj.201694700

36. Ritchie DL, Ironside JW (2017) Neuropathology of human prion diseases. Prog Mol Biol Transl Sci 150:319-339. https://doi.org/10.1016/bs.pmbts.2017.06.011

37. Safar JG, Geschwind MD, Deering C, Didorenko S, Sattavat M, Sanchez H, Serban A, Vey M, Baron H, Giles K et al (2005) Diagnosis of human prion disease. Proceedings of the National Academy of Sciences of the United States of America, vol 102, pp 3501-3506. https:/doi.org/10.1073/pnas.0409651102

38. Salick MR, Wells MF, Eggan K, Kaykas A (2017) Modelling Zika virus infection of the developing human brain in vitro using stem cell derived cerebral organoids. J Visualized Exp JoVE. https://doi.org/10.3791/56404

39. Sikorska B, Knight R, Ironside JW, Liberski PP (2012) Creutzfeldt-Jakob disease. Adv Exp Med Biol 724:76-90. https://doi.org/10.1007/978-1-4614-0653-2_6

40. Sloan SA, Andersen J, Pasca AM, Birey F, Pasca SP (2018) Generation and assembly of human brain region-specific three-dimensional cultures. Nat Protoc 13:2062-2085. https://doi.org/10.1038/s41596-018-0032-7

41. Victoria GS, Arkhipenko A, Zhu S, Syan S, Zurzolo C (2016) Astrocyte-toneuron intercellular prion transfer is mediated by cell-cell contact. Sci Rep 6 : 20762. https://doi.org/10.1038/srep20762

42. Wilham JM, Orrú CD, Bessen RA, Atarashi R, Sano K, Race B, Meade-White KD, Taubner LM, Timmes A, Caughey B (2010) Rapid end-point quantitation of prion seeding activity with sensitivity comparable to bioassays. PLoS Pathog 6:e1001217. https://doi.org/10.1371/journal.ppat.1001217

43. Xiang Y, Tanaka Y, Patterson B, Kang YJ, Govindaiah G, Roselaar N, Cakir B, Kim KY, Lombroso AP, Hwang SM et al (2017) Fusion of Regionally Specified hPSC-Derived Organoids Models Human Brain Development and Interneuron Migration. Cell stem cell 21:383-398.e387. https://doi.org/10. 1016/j.stem.2017.07.007

44. Yakoub AM (2019) Cerebral organoids exhibit mature neurons and astrocytes and recapitulate electrophysiological activity of the human brain. Neural Regen Res 14:757-761. https://doi.org/10.4103/1673-5374.249283

45. Zamanian JL, Xu L, Foo LC, Nouri N, Zhou L, Giffard RG, Barres BA (2012) Genomic analysis of reactive astrogliosis. J Neurosci 32:6391-6410. https:// doi.org/10.1523/jneurosci.6221-11.2012

46. Zhang Y, Chen K, Sloan SA, Bennett ML, Scholze AR, O'Keeffe S, Phatnani HP, Guarnieri P, Caneda C, Ruderisch N et al (2014) An RNA-sequencing transcriptome and splicing database of glia, neurons, and vascular cells of the cerebral cortex. J Neurosci 34:11929-11947. https://doi.org/10.1523/ jneurosci.1860-14.2014

47. Zhu S, Victoria GS, Marzo L, Ghosh R, Zurzolo C (2015) Prion aggregates transfer through tunneling nanotubes in endocytic vesicles. Prion 9:125-135. https:/doi.org/10.1080/19336896.2015.1025189 